\title{
Atividade da consulta de Medicina Geral e Familiar: a avaliação do seu impacto pelos seus utilizadores
}

\author{
Medical services in the field of family health: \\ impact assessment from the users perspective
}

Luiz Miguel Santiago*

\section{Resumo}

O conhecimento do impacto da atividade de Medicina Geral e Familiar é imprescindível, permitindo a retroação corretora, e o conhecimento do que pensam destas consultas os seus utilizadores é algo ainda não estudado. O objetivo do estudo é medir o impacto sentido pelos Utilizadores de Consulta de Medicina Geral e Familiar - especificamente avaliar tal em função do sexo, da idade, da formação e da atividade profissional dos utilizadores. Foram realizados: estudo transversal com intenção analítica; aplicação de duas perguntas do MOS-SF 36 a utentes de um Centro de Saúde nos dias 14 e 15 de Março de 2007; amostra de conveniência em maiores de 16 anos, em função da ordem de inscrição na consulta do médico. Foram recebidos 127 questionários (57,7\%) dos 220 entregues. A idade média obtida foi 50,4

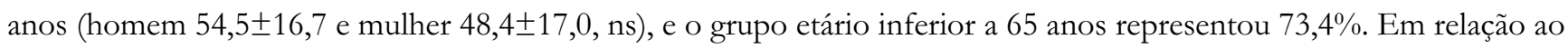
ano anterior, foram obtidas saúde igual ou superior a boa para 34,6\% e saúde superior a um pouco melhor para $22,2 \%$. O estudo discute ainda que o conhecimento do que os utilizadores pensam sobre a atividade do médico é importante para potenciar o resultado de cada contato, assim melhorando a acessibilidade. Os resultados encontrados não têm comparação com outros estudos, e a conclusão foi que a qualidade do estado de saúde, comparada ao ano anterior, revela ser melhor para 22,2\% dos respondedores, contra 19,1\%, que, julga-se, apresentarem estado pior.

\begin{abstract}
Assessing the impact of the family health services is indispensable for a corrective feedback, however it has not been studied at yet how the users feel about these services. The objective of this study is thus to measure the impact of the family health services from the viewpoint of the users, specifically according to sex, age, educational level and professional activity. The survey carried out with this purpose included: a transversal analytical study; application of two MOS SF 36 questionnaires to the users of a family health unit on March 14 and 15, 2007; convenience sample of patients with more than 16 years of age, following the order of appointments. One hundred and twenty seven (57,7\%)

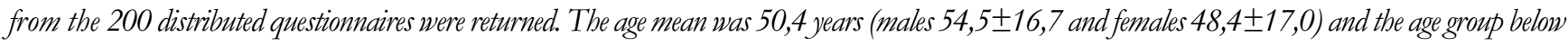
65 years represented 73,4\%. In comparison to the year before, 34,6\% ranked their health status as equal, better or good and 22,2\% as a little better. The study still argues that knowing what the users think about the doctor's work is important for increasing the effectiveness of each appointment, thus improving the accessibility. There are no data available for comparing the results found in this study and it is concluded that, in comparison to the year before, the health status of 22,2\% of users was found better against $91,1 \%$ qualifying their health status as worse.
\end{abstract}

Palavras-chave: Garantia de Qualidade em Saúde; Qualidade de Vida; Medicina de Família e Comunidade.
Key Words: Quality Assurance, Health Care; Quality of Life; Family Practice.

* Médico, Doutorando da Faculdade de Medicina da Universidade de Coimbra, especialista em Medicina Geral e Familiar, Centro de Saúde de Eiras, sub-região de Saúde de Coimbra, Portugal. 


\section{Introdução}

No âmbito da avaliação da qualidade ${ }^{1,2,3}$, a prática da Medicina, em particular a de Clínica Geral/Medicina Familiar (MGF), deve ser medida de acordo com a situação e o contexto que a conhecemos em Portugal, segundo a organização de um Centro de Saúde ${ }^{3,4,5}:$ a diversidade de apresentação das situações que se apresentam aos médicos especialistas, pois que esta especialidade médica lida com indivíduos que procuram um médico para alívio das suas queixas - quer de doença, quer apenas de seu temor ${ }^{4,6,7}$.

Alguns trabalhos realizados em outros contextos socioprofissionais demonstram que a qualidade pode ser medida pelos utilizadores em áreas muito particulares, como a medicação ${ }^{8,9,10}$. Trabalho publicado na área geográfica do Distrito de Coimbra versou sobre a temática da qualidade estrutural de uma forma conceptual, tentando verificar a existência das condições necessárias para a prática de Consulta ${ }^{11}$.

Há especificidades próprias de estrutura, de processo e de resultado que são já medidas pelos decisores de saúde ${ }^{12} \mathrm{e}$ há resultados de inquéritos que fazem pensar que os médicos de Clínica Geral/Medicina Geral e Familiar em Portugal estão intrinsecamente e explicitamente voltados para a qualidade ao pretenderem ter artigos que reflitam boas-práticas ${ }^{13}$.

No entanto, falta ainda medir qual a qualidade sentida pelos utilizadores, medida pelo seu julgamento quanto ao estado comparativo com o, atualmente, sentido ${ }^{14}$.

Talvez, assim se possa gerar um clima de automotivação para a qualidade de trabalho em que o médico e a estrutura em que trabalha estejam em permanente mutação, evitando a insatisfação que pode advir do hábito da prática ${ }^{15}$.

É necessário criar cultura de cuidados centrados no doente - entendido este como uma das partes da equação saúde -, sendo um dos pontos integrantes da resposta ao problema "doença"16,17, pelo que se julga importante realizar trabalho na área da qualidade, em uma estrutura de prestação de cuidados de saúde primários, segundo um modelo simples de abordagem.

\section{Objetivos}

Medir o impacto sentido pelos utilizadores de consulta de Medicina Geral e Familiar - especificamente, avaliar tal em função do sexo, da formação e da atividade profissional dos utilizadores.

\section{Material e métodos \\ Material}

- Utilizadores da Consulta de Clínica Geral de um Centro de Saúde.

- Formulário com duas questões do MOS-SF $36^{14}$.

- Funcionários de um Centro de Saúde que distribuíram formulário com as perguntas.

- Base de dados em SPSS, versão 11.0.

\section{Metodologia}

Estudo transversal, com intenção analítica, realizado em março de 2007.

População escolhida por amostragem de conveniência de indivíduos com idade igual ou superior a 16 anos, que, em tais dias, foram à consulta do seu médico e tendo freqüentado a consulta no primeiro trimestre do ano anterior. $\mathrm{O}$ formulário, contido em envelope endereçado, foi distribuído pelos funcionários administrativos, por ordem seqüencial de inscri- ção na consulta até o limite de formulários disponíveis por médico, em função da produção relativa de consultas em semana de fevereiro de 2007. Foi pedido aos usuários, pelo funcionário administrativo da consulta, que preenchessem o formulário e o colocassem, dobrado no envelope, em caixa própria na sala de espera à saída da consulta. Foi garantido sigilo e confidencialidade. A informação dada pelos funcionários foi previamente discutida com estes, tendo havido ação de formação sobre a uniformização desta atividade. O limite de 220 formulários deveu-se a questões orçamentais.

A população foi caracterizada em termos de sexo, formação e atividade profissional, em função dos registos informáticos de inscrição na consulta, sendo critério de inclusão saber ler e escrever.

Os dados foram inseridos em ficheiro informático 
SPSS versão 11.0. O trabalho estatístico descritivo e inferencial utilizou o teste $t$ de student para variáveis numéricas e o $\chi^{2}$ para variáveis ordinais.

\section{Resultados}

A população foi constituída por 220 inscritos, que compareceram às consultas em 14 e 15 de março de 2007, sendo $n=61(27,7 \%)$ do sexo masculino, com uma idade média de 33,9 anos (mulher 27,3 anos e homem 58,5 anos, $\mathrm{p}=0,000)$, segundo o estudo populacional realizado pelo Gabinete SINUS (Serviço de Informação sobre Utentes da Saúde) da autoridade de saúde do Distrito de Coimbra. Desta população, os inscritos e freqüentadores da consulta, 108 (48,8\%) tinham menos de 65 anos.

A amostra consta de 127 formulários, representando $57,7 \%$ dos 220 entregues, e apresenta: 43 homens (34,1\%), sendo a idade média geral de 50,4 $\pm 17,0$ anos (homem

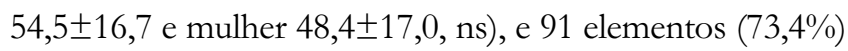
representaram o grupo etário inferior a 65 anos.

Na Tabela I, é referida a distribuição na amostra

Tabela I. Distribuição da formação académica na amostra

\begin{tabular}{|c|c|c|c|c|c|}
\hline Formação $(*)(* *)$ & $\begin{array}{l}\text { Total } \\
\text { n ( } \%)\end{array}$ & $\begin{array}{l}\text { Homens } \\
\text { n (\%) (*) }\end{array}$ & $\begin{array}{l}\text { Mulheres } \\
\text { n (\%)(*) }\end{array}$ & $\begin{array}{l}</=65 \\
\mathrm{n}(\%)(* *)\end{array}$ & $\begin{array}{l}>/=65 \\
\mathrm{n}(\%)(* *)\end{array}$ \\
\hline Sabe ler e escrever & $6(1,4)$ & $11(13,4)$ & $5(5,6)$ & $11(33,3)$ & $17(13,5 \%)$ \\
\hline $4^{a}$ classe & $27(62,8)$ & $46(56,1)$ & $50(55,6)$ & $22(66,7)$ & $74(58,7)$ \\
\hline $12^{\circ}$ ano & $5(1,2)$ & $11(13,4)$ & $16(17,8)$ & - & $16(12,7 \%)$ \\
\hline Técnica & - & $3(3,7)$ & $3(3,3)$ & - & $3(2,4 \%)$ \\
\hline Superior & $5(1,2)$ & $11(13,4)$ & $16(17,8)$ & - & $16(12,7)$ \\
\hline Total & 43 & 82 & 90 & 33 & 126 \\
\hline
\end{tabular}

Nota: (*) ns na análise por sexos. $\left(^{* *}\right) p=0,000$ por grupo etário $<65$ ou $>66$ anos, sendo mais baixa a formação no grupo mais idoso.

Tabela II. Atividade profissional da amostra

\begin{tabular}{|c|c|c|c|c|c|}
\hline $\begin{array}{l}\text { Atividade } \\
\text { Profissional }(*)(* *)\end{array}$ & $\begin{array}{l}\text { Total } \\
\text { N }(\%) n\end{array}$ & $\begin{array}{l}</=65 \\
\ln (\%)(*)\end{array}$ & $\begin{array}{l}>65 \\
\mathrm{n}(\%)(*)\end{array}$ & $\begin{array}{l}\text { Homens } \\
\mathrm{n}(\%)(* *)\end{array}$ & $\begin{array}{l}\text { Mulheres } \\
\text { n }(\%)(* *)\end{array}$ \\
\hline Rural & $1(1,1)$ & $1(3,0)$ & $1(2,4)$ & $2(2,5)$ & $3(2,4)$ \\
\hline Comércio & $10(11,5)$ & - & $2(4,9)$ & $8(9,9)$ & $10(8,1)$ \\
\hline Indústria & $9(10,3)$ & $1(3,0)$ & $5(12,2)$ & $5(6,2)$ & $10(8,1)$ \\
\hline Serviços & $34(39,0)$ & - & $8(19,5)$ & $26(21,1)$ & $34(27,6)$ \\
\hline Doméstica & $8(9,2)$ & $5(15,2)$ & - & $14(17,3)$ & $14(11,4)$ \\
\hline Desempregado & $7(8,0)$ & - & $5(12,2)$ & $3(3,7)$ & $8(6,5)$ \\
\hline Reformado & $13(14,9)$ & $26(68,8)$ & $18(43,9)$ & $20(24,7)$ & $39(31,7)$ \\
\hline Estudante & $5(5,7)$ & - & $2(4,9)$ & $3(3,7)$ & $5(4,1)$ \\
\hline Total & 87 & 33 & 41 & 81 & 123 \\
\hline
\end{tabular}

Nota: Homens ${ }^{2}\left(^{*}\right) p=0,000 ;\left(^{* *}\right) p=0,018$ para a distribuição por sexos (homem e com comparativa maior percentagem de reformados). 
dos vários tipos de formação considerados, valendo realçar que 27,8\% têm formação acadêmica avançada.

$\mathrm{Na}$ Tabela II, referimos a distribuição da atividade profissional na amostra.

$\mathrm{Na}$ Tabela III, fornecemos os resultados obtidos nas respostas às duas afirmações do SF $36^{14}$. Nota-se que, para a distribuição por tipo de formação, a percentagem de respondentes em estado de saúde Razoável/Mau é de 73,6\%,

\section{Discussão}

Trata-se de um estudo de características epidemiológicas e fundamentado em população de escolha pelo método de conveniência. De fato, o autor dispunha apenas de um concreto número de formulários, tendo solicitado a sua aplicação à população que soubesse ler e escrever e que fosse maior de 16 anos de idade, nos dias especificamente escolhidos - e apenas divulgados no próprio dia -, e que ti-

Tabela III. Resposta às duas afirmações do SF 36 acerca do estado de saúde presente e em comparação com o ano transato

\begin{tabular}{|c|c|c|c|c|c|}
\hline 1. Em geral diria que a & Excelente & Muito Boa & Boa & Razoável & Má \\
\hline $\begin{array}{l}\text { sua saúde está: } \\
\mathrm{n}=127(*, * *, * * *, * * * *)\end{array}$ & $5,5 \%$ & $7,1 \%$ & $22,0 \%$ & $53,5 \%$ & $11,8 \%$ \\
\hline $\begin{array}{l}\text { 2. Em comparação ao } \\
\text { ano passado diria que a } \\
\text { sua saúde está: } \\
\mathrm{n}=126(*, * *, * * *, * * * *)\end{array}$ & $\begin{array}{c}\text { Muito melhor } \\
6,3 \%\end{array}$ & $\begin{array}{l}\text { Um pouco } \\
\text { melhor } \\
15,9 \%\end{array}$ & $\begin{array}{l}\text { Igual } \\
58,7 \%\end{array}$ & $\begin{array}{c}\text { Um pouco } \\
\text { pior } \\
15,1 \%\end{array}$ & $\begin{array}{c}\text { Muito pior } \\
4,0 \%\end{array}$ \\
\hline
\end{tabular}

Nota: utilizando o $X^{2}$ Por grupo etário (*): ns para ambas as questões. / Por sexos (**): ns para as duas questões. / Por tipo de formação (***): $p=0,011$ para afirmação 1 e ns para afirmação 2. / Por tipo de atividade profissional (****): ns para ambas as afirmaçöes.

sendo 45,7\% para aqueles com formação superior ao $12^{\circ}$ ano, $5,5 \%$ os que têm formação inferior a $12^{\circ}$ ano e estado de saúde excelente ou muito melhor, contra $31,4 \%$ de respondentes em tal sentimento, mas com formação superior a $12^{\circ}$ ano. O bom estado de saúde tem freqüências de 20,9\% e $22,9 \%$ para a formação menor ou maior que $12^{\circ}$ ano.

A evolução sentida no estado de saúde em função do estado de saúde atual foi avaliada, valendo destacar que quem mais evoluiu no sentido da piora, segundo o que podemos verificar na Tabela IV, são aqueles que pior estado atual têm. vessem freqüentado a consulta do seu médico no primeiro trimestre de 2006. Dada a necessidade de abrangência populacional em um Centro de Saúde que tem 12 médicos atendendo uma população inscrita de 16.500 pessoas, optou-se pela entrega de formulários, em envelope endereçado, a todos os que em tais dias se inscrevessem na consulta segundo um rateio por médico em função da produção de consultas em fevereiro de 2007.

O instrumento utilizado para a avaliação do presente estudo foi já alvo de validação e tradução para o português ${ }^{14}$. Admitimos viéses de percepção, de memória e de

\begin{tabular}{|c|c|c|c|c|c|}
\hline $\begin{array}{l}\text { 1. Estado de saúde atual / } \\
\text { estado de saúde no ano } \\
\text { passado }(*)\end{array}$ & $\begin{array}{c}\text { Excelente } \\
\mathrm{n}(\%)\end{array}$ & $\begin{array}{c}\text { Muito Bom } \\
\mathrm{n}(\%)\end{array}$ & $\begin{array}{l}\text { Bom } \\
\mathrm{n}(\%)\end{array}$ & $\begin{array}{c}\text { Razoável } \\
\mathrm{n}(\%)\end{array}$ & $\begin{array}{l}\text { Mau } \\
\mathrm{n}(\%)\end{array}$ \\
\hline Muito Melhor & $4(57,1)$ & - & $1(3,6)$ & $3(4,4)$ & - \\
\hline Um pouco melhor & - & $2(22,2)$ & $5(17,9)$ & $12(17,6)$ & $1(7,1)$ \\
\hline Igual & $3(42,9)$ & $7(77,8)$ & $21(75,0)$ & $35(51,5)$ & $8(57,1)$ \\
\hline Um pouco pior & - & - & $1(3,6)$ & $17(25,0)$ & $1(7,1)$ \\
\hline Muito pior & - & - & - & $1(1,5)$ & $4(28,6)$ \\
\hline
\end{tabular}

Nota $(*) p=0,000\left(X^{2}\right)$ 
intenção no preenchimento do formulário. Fatores diversos e aqui não estudados, como o tempo de espera para a efetivação da consulta ou o resultado da presente, podem ter influenciado os resultados.

Quanto à amostra, podemos dizer que é particularmente jovem, com uma formação sobretudo básica e com predomínio de atividade profissional nos serviços, constituindo o sexo masculino $34,1 \%$ - o que espelha a população.

Em Medicina de Família e Comunidade (em Portugal, designada por Medicina Geral e Familiar), é importante a percepção do estado de saúde dos pacientes e mesmo o conhecimento do resultado da consulta médica em todas as suas vertentes, dado o conjunto de sinais, sintomas e patologias apresentadas pelos pacientes ${ }^{18}$. Aspectos tão simples como a duração da consulta podem fazer alterar a percepção do estado de saúde ${ }^{19}$.

Não tendo verificado a existência de qualquer trabalho que reflita o aqui objetivado parece poder dizer-se que a atividade do corpo clínico deste Centro de Saúde, em conjunto com todos aqueles que com eles colaboram, tem vindo a produzir serviços com a qualidade suficiente para os resultados obtidos. Em outros contextos podem ser feitas diferentes avaliações dependentes por exemplo dos registos efetuados ${ }^{20}$, sendo sempre necessário o desenho e a otimização da utilização de ferramentas de análise e de equipes destinadas à função da medição da qualidade, quer na óptica da estrutura que trabalha segundo um processo definido e obtém resultados, quer na óptica dos seus utilizadores ${ }^{21}$.

Não pode, no entanto, deixar de ser refletido que é naqueles que pior se sentem neste momento de observação que houve pior evolução do estado de saúde e que é no grupo etário mais elevado e naqueles que têm menor freqüência escolar que o estado de saúde parece ter mais piorado. A razão para tal precisa, agora, ser estudada.

\section{Conclusões}

Medir o impacto sentido pelos utilizadores de consulta de Medicina Geral e Familiar - especificamente avaliar tal em função do sexo, da formação e da atividade profissional dos usuários.

A medição do impacto da atividade de um Centro de Saúde, julgado pelos seus usuários, revela que, para $34,1 \%$, o estado de saúde atual é igual ou superior a bom, estando igual ao ano transato para 58,7\% e melhor para $22,2 \%$. Para 19,1\%, o estado de saúde está pior que no ano anterior, sobretudo para os mais idosos e aqueles que têm menor formação acadêmica.

\section{Referências}

1.Donabedian A. The definition of quality and approach to its assessment. Ann Harbour: Health Administration Press; 1980.Vol.1.

2.Donabedian A. The methods and findings of quality assessment and monitoring: an illustrated analisys. Ann Harbour: Health Administartion Press; 1985. Vol.3.

3.Ferreira PL, Raposo V. A governação em saúde e a utilização de indicadores de satisfação. Rev Port Clin Geral 2006;22:285-296

4. Disponível em: http://fnam.pt/smzc/ficheiros/ Dec_Lei_73_90.pdf.

5. Decreto-Lei n. ${ }^{\circ}$ 157/99, de 10 de Maio

6. A definição Europeia de Medicina Geral e Familiar. Versão Reduzida - EURACT. Rev Port Clin Geral 2005; 21:511-516

7. Disponível em http://www.mcsp.min-saude.pt/NR/ rdonlyres/EB139DE8-00B1-4B54-A19F-418D98D52DF0/ 2824/linhas_acção.pdf

8.Miranda JÁ. O que esperam os nossos clientes? Rev Port Clin Geral 2001;17:11-12

9.Ferreira PL. Avaliação dos doentes de cuidados primários: aspectos da clínica geral mais importantes para os doentes. Rev Port Clin Geral 2001;17:15-45

10. Santiago LM, Cobrado NM. Custos Directos da Terapêutica farmacológica no ambulatório de Clínica Geral. Rev Port Clin Geral. 2002;18:351-9

11. Neto MG, Jacob IM, Santiago LM. Cuidados de saúde ao Idoso: avaliação da qualidade dos serviços. Espelho, Sub-região de Saúde de Coimbra; 1997. v.6

12. Disponível em: http://www.mcsp.min-saude.pt/NR/ 
rdonlyres/EB139DE8-00B1-4B54-A19F-418D98D52DF0/

3769/IndicadoresUSFMCSP20060412.pdf

13. Maria VA. Satisfação com a Revista: inquérito aos leitores. Rev Port Clin Geral. 2001;17:95

14. Criação da versão portuguesa do Medical Outcome Study Short Forum 36 (MOS SF36), Parte II: Testes de validação. Ferreira PL. Acta Med Port. 2000;13(3):119-127. 15. Ferreira PL. Avaliação dos doentes de cuidados primários. Rev Port Clin Geral. 2000; 16:53-62.

16. Hespanhol A, Costa Pereira A, Sousa Pinto A. Insatisfação Profissional em Medicina Geral e Familiar: um problema intrínseco dos médicos ou das condições de trabalho? Rev Port Clin Geral. 2000;16:183-199

17. Ferreira PL, Raposo V, Godinho P. A voz dos utilizadores dos Centros de Saúde. Lisboa: Ministério da Saúde; 2006:7584.

18. Santos I. A parte de leão da Medicina Geral e Familiar. Rev Port Clin Geral. 2007;23:175-178.

19. Cannon B. General practice consultations: how well do doctors predict patient satisfaction? Aust Fam Physician. 36:3:185-187.

20. Rao M, Clarke A, Sanderson C, Hammersley R. Avaliação pelos próprios doentes da qualidade dos cuidados primários em comparação com medidas objectivas baseadas nos processos clínicos da qualidade técnica dos cuidados: estudo transversal. BMJ. 2006; 333: 19-22.

21. Miralles JD. Plano para a melhoria contínua da qualidade: os projectos de melhoria - aspectos práticos da qualidade assistencial em Cuidados de Saúde Primários. Postgraduate Medicine. 2007; 27(2): 54-62

\section{Endereço para correspondência}

Luiz Miguel Santiago

Quinta de Voimarães, lote $12-5^{\circ} \mathrm{D}$

3000-377

Coimbra - Portugal

\section{Endereço eletrônico}

lmsantiago@netcabo.pt 